\title{
Rapidly Developing Obstructive Sleep Apnea due to Fixation Failure of Atrophic Mandibular Bilateral Body Fractures
}

\author{
${ }^{1}$ Ankit Arora, ${ }^{2}$ Sahil Gagnani, ${ }^{3}$ Gaurav Jain, ${ }^{4}$ Rahul Yadav
}

\begin{abstract}
Introduction: The aim of this report was to present a case of obstructive sleep apnea (OSA) in a 74-year-old male secondary to $2 \mathrm{~mm}$ miniplates fixation failure.

Method: The patient had a preoperative apnea/hypopnea index of 21 and was successfully treated by $2.4 \mathrm{~mm}$ reconstruction plate for bifocal mandibular fracture and thus treating OSA.
\end{abstract}

Results: At two years follow-up, the patient has been doing well with no signs and symptoms of OSA.

Discussion: Treatment of atrophic mandibular fractures can certainly be challenging. Elderly, infirm patients, unopposed muscle pull, diminished blood supply, bone inadequate qualitatively and quantitatively for osteosynthesis, and inadequate ridges for stable splints or prostheses all lead to treatment that is difficult and prone to failure. Bifocal fractures that involve the anterior dentate region of the mandible may cause the lingual displacement of the central proximal fragment. Therefore, they can epitomize an important risk for the upper airways.

Bilateral body fracture in an edentulous mandible as a cause of OSA has never been reported in the literature. Here, we present such a case in a 74-years-old male patient who had a preoperative apnea/hypopnea index of 21 following the failed fixation from the original operation.

Keywords: Atrophic mandible, Bilateral parasymphyseal fracture, Obstructive sleep apnea, Reconstruction.

How to cite this article: Arora A, Gagnani S, Jain G, Yadav R. Rapidly Developing Obstructive Sleep Apnea due to Fixation Failure of Atrophic Mandibular Bilateral Body Fractures. Indian Sleep Med 2018;13(1):18-20.

Source of support: Nil

Conflict of interest: None

\section{INTRODUCTION}

Obstructive sleep apnoea/hypopnea syndrome (OSAHS)

\footnotetext{
${ }^{1-3}$ Ex-Senior Resident, ${ }^{4}$ Assistant Professor

${ }^{1-4}$ Center for Dental Education and Research (CDER), Division of Oral and Maxillofacial Surgery, All India Institute of Medical Sciences, New Delhi, India
}

Corresponding Author: Rahul Yadav, Assistant Professor, Centre for Dental Education and Research (CDER), Division of Oral and Maxillofacial Surgery, All India Institute of Medical Sciences, New Delhi, India, Tel: +91 9999994578, e-mail: drrahulomfs@gmail.com is the sporadic reduction (hypopnea) or cessation (apnoea) of airflow during sleep that results from pharyngeal narrowing or collapses. ${ }^{1}$ Risk elements may encompass obesity, male gender, retrognathia, hypothyroidism, nasal obstruction and evening alcohol ingestion (ASDA 1996). ${ }^{2}$ The severity of sleep apnoea is commonly measured by polysomnography, incorporating measurements of the frequency of apnea-hypopnea index (AHI), reducing $\mathrm{AHI}$ and diminishing the catastrophe of sleep apnoea in terms of symptoms and quality of life are the constitutional intention of therapy (SIGN 2003). ${ }^{3}$

Fractures of the severely atrophic $(<10 \mathrm{~mm})$ edentulous mandible are not prevalent, representing less than 1 to $5 \%$ of all mandibular fractures. ${ }^{4}$ Fractures of the atrophic and edentulous (toothless) mandible (lower jaw) are fairly common in the elderly population. Atrophy, where the mandible is $20 \mathrm{~mm}$ or less in vertical height, and weakening of the edentulous mandible which tends to crop up as a consequence of diminished vascularity and abate blood flow. Conservative options that can aid in stabilizing the fracture encompass the use of dentures, Gunning splints, and external fixation appliances, but these procedures may not be pertinent for grossly displaced edentulous fractures of the mandible. ${ }^{5}$ Sporadically, more invasive management may be appropriate with open reduction and fixation with mini plates, reconstruction plates, rigid fixation-bone plating techniques, compression plates, supraperiosteal paraskeletal clamp-on bone plates (Mennen plates) and which may encompass the use of autogenous bone grafts and primary bone or bone marrow grafting. ${ }^{6}$ Inflammatory complications may cultivate more often than in other age groups, and there is a higher proportion of non-union with severely atrophied mandibles.

Anterior bifocal bilateral mandibular body fractures may embroil a challenging management because they can compromise the upper airway, and they can hamper pertinent osseous reduction. Rigorous reduction and internal fixation of these fractures have been critical to restoring form and function of the mandible. The genioglossus is crucial in maintaining a patent pharyngeal airway by anterior translation of the tongue. It serves as a preeminent determinant of tongue position. Bilateral fractures 
of the anterior mandible may cause lingual deracination of the central distal fragment culminating in obstructive sleep apnoea.

\section{Case report}

A 74-year-old male reported to the oral and maxillofacial surgery department with complaints of disturbed sleep with repeated awakening for 2 months which was deliberately increasing in severity. Lately, he was also experiencing inordinate daytime sleepiness, tiredness, and fatigue which restricted his periodic activities. According to his family members, he was increasingly snoring during sleep, and there was also a perceptible transition in his facial profile by virtue of

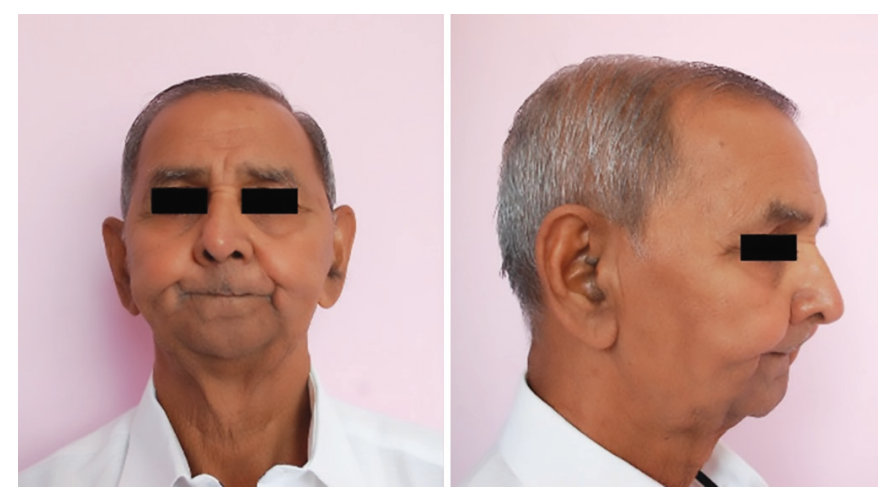

Fig. 1: Frontal and lateral profile showing posteriorly displaced chin.

piecemeal depression of his chin (Figure 1). Family history was unremarkable. The patient had no recent history of weight gain. His weight was $71 \mathrm{~kg}$ with a body mass index (BMI) of 24.4. Neck diameter was $33 \mathrm{~cm}$. He had been treated for fracture mandible due to trauma four months back. Radiographic investigations showed a fracture of
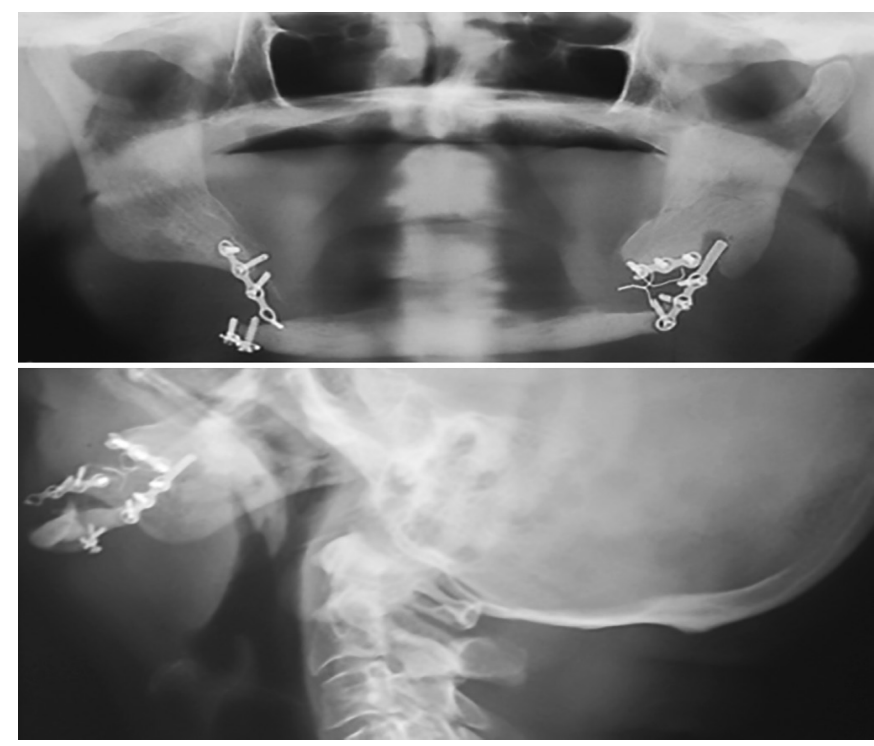

Fig. 2: Orthopantomogram and lateral skull view showing fixation failure of mandibular fracture with displaced anterior mandibular segment. mandibular fixation miniplates bilaterally (Figure 2). An overnight polysomnography study revealed a moderate OSA with apnea-hypopnea index (AHI) of 21 with an average oxygen saturation of $97 \%$ and minimum oxygen saturation of $90 \%$. The diagnosis of moderate obstructive sleep apnea (OSA) due to fracture of mandibular fixation was made. Under general anesthesia, the fracture was reduced and fixed with a long-spanning $2.4 \mathrm{~mm}$ reconstruction plate which relieved him of his symptoms and thus treating OSA (Figure 3). The patient reported no overt sleep apnea symptoms with postoperative overnight polysomnography study revealing $\mathrm{AHI}$ index of 0 and an average oxygen saturation of $100 \%$.

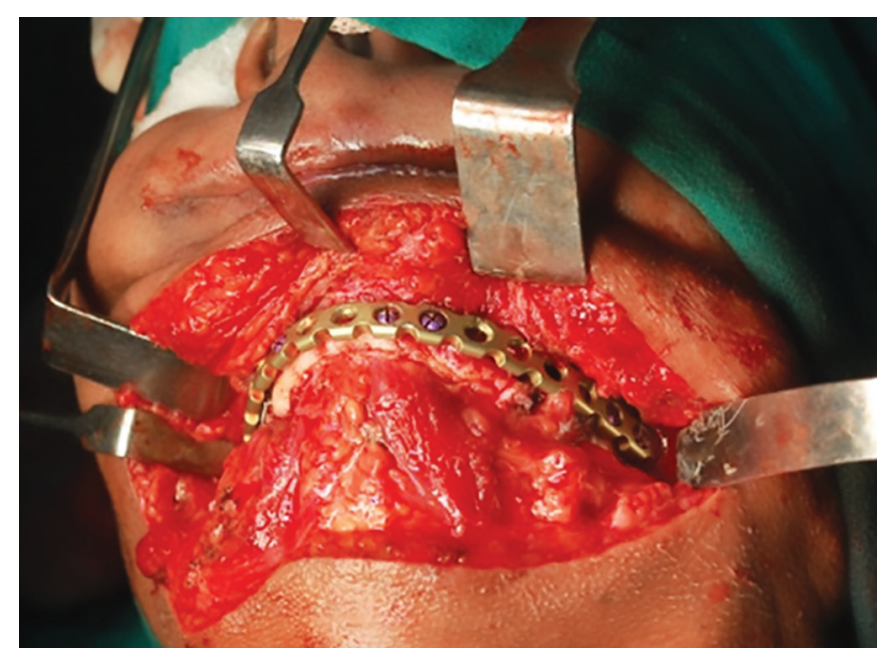

Fig. 3: Intraoperative view with mandible reconstructed with 2.4 $\mathrm{mm}$ reconstruction plate.

\section{DISCUSSION}

OSA is caused by abeyance of airflow secondary to collapse of the upper airway during sleep. Airway patency is mainly maintained by two conflicting forces: the soft tissues encircling the airway which gravitate to collapse it and the activity of the pharyngeal dilator muscles which maintains it during sleep. The present case was peculiar as OSA was of recent onset with a short history of two months and no evidence of exorbitant obesity or increased neck diameter. There was upper airway compromise on account of the failure of previously fixed bilateral parasymphyseal fracture of the mandible. It is acknowledged that an edentulous mandible steadily loses its vertical height with long-term use of complete dentures. For this patient, the original surgery provided inadequate fixation resulting in the significant displacement of the distal mandibular segment with a gradual downward and backward displacement created by the pull of the genioglossus and geniohyoid muscles which created the OSA symptoms in a relatively short course of time. Due to preceding inadequate fixation (use of miniplates), there was a failure of fixation apparatus in 
our patient. In the absence of equitable fixation, there was a gradual downward and backward displacement of the fractured fragment by the pull of genioglossus and geniohyoid muscles which lead to features of OSA in a short course of time. While OSA is diagnosed usually by a sleep specialist, our case demonstrates a bizarre emergency presentation of this disorder.

OSA is clinically characterized by escalation in the frequency and ferocity of snoring, incessant awakenings and restless sleep. An emblematic obstructive episode consists of outright cessation of respiration for at least ten seconds with the absence of snoring followed by awakening and a loud snort leading to hyperventilation. The high degree of respiratory effort required for inhalation against the collapsed pharynx is the reasonable cause of awakening in such patients. Once awake, the pharyngeal dilator activity is restored, and airway patency is maintained. Overnight polysomnography is considered the gold standard in the diagnosis. ${ }^{7}$

In atrophic mandible fracture, few surgeons proponents the use of miniplates. The premise behind the use of this type of plate is "the smaller the better;" the concept being that a small plate is ample for the purpose and, because it is small, is less feasible to result in periosteal stripping than a larger plate. However, Eyrich noted that miniplates are accountable for failure due to the ineptitude of the plates to thwart the load placed on them by the maxillomandibular forces. ${ }^{8}$ There is only one speculative justification for miniplates failure in this case. Champy's humanistic works on the biomechanics of the mandible validated that there are two trajectories of neutral force between the mental foramen as the mastication forces cause torsional contortion of the mandible. Thus, a frontal fragment of the mandible fixed in three dimensions by two titanium miniplates presumably changes the biomechanics in this area, increasing the blunt force on the anterior alveolus of the mandible. ${ }^{9}$

A reconstruction plate, because of its capability to cater primary stability, is the AO/ASIF (Arbeitsgemeinschaft fur Osteosynthesefragen/Association for the Study of Internal Fixation) technique of treating an atrophic edentulous mandible fracture. At high loads, atrophic mandibles (10 $\mathrm{mm}$ in height) repaired with reconstruction plates equip resistance to displacement equivalent to that found in mandibles $40 \mathrm{~mm}$ in height repaired with miniplates, may be less prone to fatigue and may show a diminished propensity for screws to alleviate under

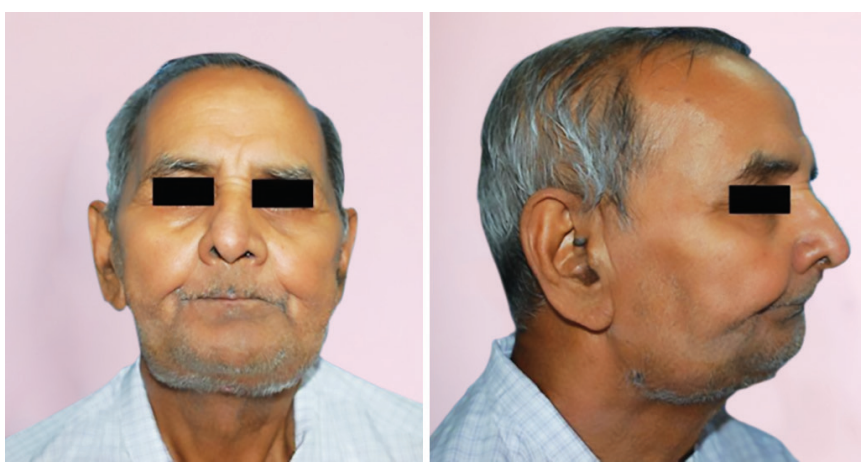

Fig. 4: Frontal and lateral profile at 2 years follow-up period.

cyclic loading, which decreases the rate of postoperative infection and failure. ${ }^{10}$ Thus, it was pronounced to operate the mandible with reconstruction plate. At two years follow up, the patient has been doing well with no signs and symptoms of OSA or mandible fracture (Figure 4).

\section{REFERENCES}

1. Sundaram S, Lim J, Lasserson T. Surgery for obstructive sleep apnoea in adults(review). Cochrane Database Syst Rev. 2013;(2).

2. Practice parameters for the treatment of obstructive sleep apnea in adults: the efficacy of surgical modifications of the upper airway. Report of the American Sleep Disorders Association. Sleep. 1996;19(2):152-155.

3. Scottish Intercollegiate Guideline Network. Management of obstructive sleep apnoea/hypopnoea syndrome in adults(Guideline No. 73). http:/ / www.sign.ac.uk. 2003.

4. Clayman L, Rossi E. Fixation of Atrophic Edentulous Mandible Fractures by Bone Plating at the Inferior Border. J Oral Maxillofac Surg. 2012;(70):883-889.

5. Barber HD. Conservative management of the fractured atrophic edentulous mandible. J Oral Maxillofac Surg. 2001;59:789-791.

6. Basa S, Uner E. Titanium mesh and autogenous iliac bone graft: treatment of a mandibular fracture delayed for one year. J Marmara Univ Dent Fac. 1997;2(4):628-630.

7. Lee NR. Evaluation of the obstructive sleep apnea patient and management of snoring. Oral Maxillofac Surg Clin North Am. 2009;21(4):377-387.

8. Eyrich GK, Grätz KW, Sailer HF. Surgical treatment of fractures of the edentulous mandible. J Oral Maxillofac Surg. 1997;55(10):1081-1087; discussion 1087-1088.

9. Rene' FOLTA` N Jir`ina HOFFMANNOVA', Martin PRETL, Filip DONEV MV. Genioglossus advancement and hyoid myotomy in treating obstructive sleep apnoea syndrome - A follow-up study. J Craniomaxillofac Surg. 2007;35:246-251.

10. Sikes JW, Smith BR, Mukherjee DP. An in vitro study of the effect of bony buttressing on fixation strength of a fractured atrophic edentulous mandible model. J Oral Maxillofac Surg. 2000;58(1):56-61; Discussion 62. 